Comparative and International Education / Éducation Comparée et Internationale

Volume 50 | Issue 1

Décembre 2021

\title{
Les élèves doués et talentueux au Canada : une étude comparative
}

Philippe Tremblay

Université Laval

philippe.tremblay@fse.ulaval.ca

Jeanne Verreault-Tremblay

Université Laval

jeanne.verreault-tremblay.1@ulaval.ca 


\title{
Recommended Citation
}

Tremblay, P. (2021). Les élèves doués et talentueux au Canada : une étude comparative. Comparative and International

Education/Éducation comparée et internationale. 50 (1). https://doi.org/10.5206/cieeci.v50i1.13676

\section{Les élèves doués et talentueux au Canada : une étude comparative Gifted and talented students in Canada: A comparative analysis}

\author{
Philippe Tremblay, Université Laval
}

\section{Résumé}

Cet article propose une analyse des politiques provinciales et territoriales des 10 provinces et des trois territoires canadiens au regard des élèves doués et talentueux, dressant un portrait comparatif du contexte dans lequel s'inscrit leur scolarisation. Y sont analysés les définitions et les domaines de la douance ainsi que les mesures pédagogiques proposées pour ces élèves. Les résultats de cette étude documentaire montrent d'abord un fort contraste entre les provinces ne reconnaissant pas cette catégorie et celles les reconnaissant. Chez ces dernières, un consensus sur la terminologie utilisée et la définition est observé. En ce qui concerne les mesures scolaires et extrascolaires, la grande majorité des provinces propose des mesures explicitement ou implicitement destinées aux élèves doués. Toutefois, celles-ci sont plus explicites et détaillées dans les provinces reconnaissant formellement cette catégorie d'élèves.

\begin{abstract}
This article presents an analysis of the provincial and territorial policies of the 10 Canadian provinces and territories for gifted and talented students, providing a comparative picture of the context in which their schooling is based. It investigates the definitions of gift and diagnostic criteria used in identifying gifted and talented students, as well as the scholar and extracurricular measures put in place for these students. The results of this literature study first show a strong contrast between provinces that do not recognize this category and those that recognize them. For the latter, there is a consensus on the terminology used and the definition. With respect to school and extracurricular measures, the vast majority of provinces propose measures explicitly or implicitly intended for these students. However, these are more explicit and detailed in provinces explicitly recognizing this category of students.
\end{abstract}

Mots-clés : douance, éducation comparée, inclusion, classe spéciale, mesures scolaires, mesures extrascolaires

Keywords: giftedness, comparative education, inclusion, special class, instructional measures, extracurricular measures 


\section{Introduction}

Le champ de la douance a fait l'objet d'une attention accrue au cours de ces dernières années en éducation comparée (ex. : Eurydice, 2006; European Agency for Development in Special Needs Education, 2009, Kanevsky et Clelland, 2013). Si plusieurs définissent généralement les élèves doués comme ceux qui manifestent un potentiel plus élevé, plus de créativité et plus de motivation à apprendre que les autres élèves dans les classes d'âge semblable (European Agency for Development in Special Needs Education, 2009), il n'y a pas de consensus sur le sujet. Malgré cela, plusieurs auteurs soulignent l'importance de comprendre la douance comme une notion fluide qui ne doit pas être envisagée en termes de capacités génétiques ou innées, mais comme une notion multidimensionnelle (Borland, 2005; Sternberg et Davidson, 2005; Smith, 2006). En effet, la douance se manifeste sous différentes formes qui nécessitent une variété de contextes et de possibilités d'apprentissage susceptibles de saisir la totalité des capacités des élèves. Elle est ainsi vue comme un construit (Sternberg et Davidson, 2005) et, par conséquent, sa conceptualisation peut évoluer au fil du temps et de l'espace.

En ce qui concerne l'espace, au Canada, l'éducation est de juridiction provinciale et territoriale. En effet, l'Acte de l'Amérique du Nord britannique de 1867 reconnaît aux 10 provinces et aux trois territoires des compétences exclusives en ces matières. En ce sens, on ne peut parler d'un système scolaire canadien, mais plutôt de 13 systèmes scolaires canadiens. $\mathrm{Au}$ niveau canadien, il n'y a pas de définition commune de la douance entre les provinces et territoires. Les diverses caractérisations se trouvent dans les différentes lois et politiques de scolarisation des élèves doués émanant des ministères et départements de l'éducation des provinces et territoires. La nature et l'étendue des dispositions pour les élèves identifiés comme doués sont ainsi déterminées par les politiques explicites, mais également implicites (Kanevsky et Clelland, 2013). Cette situation contribue au manque d'information sur les politiques et dispositifs scolaires destinés aux élèves doués et talentueux.

Par ailleurs, on observe un manque d'études en langue française dans le domaine. De plus, des barrières linguistiques, culturelles ou politiques viennent limiter les connaissances des systèmes scolaires. Pour cette raison, il est très important de comprendre comment la notion de douance est conceptualisée dans les systèmes scolaires et comment une telle conceptualisation peut avoir une incidence sur les politiques scolaires et les mesures pédagogiques proposées.

Cet article actualise et prolonge le travail de Kanevsky et Clelland (2013) en examinant plus étroitement les politiques liées aux nombreuses pratiques et dispositifs scolaires dans chaque province et territoire. L'objectif général est de réaliser une analyse comparative des politiques et des dispositifs relatifs aux élèves doués et talentueux de chaque province et territoire canadien. Dans ce cadre, cette recherche a deux objectifs spécifiques. Le premier consiste à identifier les provinces qui ont une catégorie d'élèves doués pour y analyser les terminologies employées, les définitions utilisées et les domaines dans lesquels cette catégorie est susceptible de s'inscrire. Le second objectif est d'analyser les mesures pédagogiques mises en œuvre pour ces élèves (explicitement ou implicitement) dans l'ensemble des provinces et territoires canadiens.

\section{Contexte}

L'enseignement au Canada est de juridiction provinciale et territoriale. La constitution reconnaît des instances intermédiaires, au niveau local ou régional, qui spécifient des modes d'organisation en commissions, districts ou conseils scolaires, d'abord sur une base confessionnelle, puis depuis 1998, également sur une base linguistique. Dans les 13 juridictions scolaires (10 provinces et trois territoires), les ministères de l'Éducation sont responsables de 
l'organisation, de la gestion et de l'évaluation. Ainsi, bien que sur les structures les 10 provinces et les trois territoires canadiens présentent des similitudes, chaque système scolaire est cependant susceptible de présenter de grandes différences au regard des politiques scolaires, de financement, etc. Enfin, au Canada, l'inclusion scolaire constitue maintenant la voie privilégiée de scolarisation des élèves à besoins spécifiques (Rousseau et al., 2009; Tremblay et Belley, 2017), quoique des différences notables apparaissent entre juridictions, principalement avec le Québec où l'intégration reste encore souvent la voie favorisée (Tremblay et Belley, 2017).

Dans le cas des politiques concernant les élèves à besoins spécifiques, et plus précisément, les élèves doués et talentueux ${ }^{1}$, bien qu'ils puissent légiférer en la matière, les provinces et territoires n'y sont pas obligés. Ainsi, certaines populations peuvent être prises en compte dans une province et pas dans une autre; il en va de même avec la définition formelle de cette population, de ses critères d'identification de même que des mesures éducatives mises en œuvre pour la soutenir sur le plan scolaire. De plus, l'approche concernant l'identification des élèves à besoins spécifiques varie de province en province. Il est tout à fait possible qu'un élève soit considéré comme doué dans une province, mais pas dans une autre. Certaines préconisent une approche catégorielle (ex. : Alberta), ce qui signifie que le seul diagnostic de la douance permet une catégorisation formelle de l'élève concerné (doué ou talentueux) qui amène des obligations de mesures et de financement. Au contraire, l'approche non catégorielle priorise plutôt l'évaluation des besoins de l'élève (ex. : Québec), nonobstant un possible diagnostic, sans financement particulier associé à un élève en particulier (Westwood, 2015; Tremblay, 2021). Par ailleurs, le soutien pour la scolarisation des élèves doués tend à être cyclique (Yewchuk, 2000). Ainsi, dans les années 1990, la combinaison de coupes aux différents budgets provinciaux et territoriaux et la mise en place de politique et de pratiques inclusives ont souvent conduit à une disparition ou du moins au déclin des dispositifs scolaires destinés exclusivement aux élèves doués et talentueux (Kanevsky et Clelland, 2013).

Au niveau local, des centres de services scolaires, les conseils scolaires ou les districts scolaires, dirigent et supervisent le fonctionnement d'une division scolaire au niveau provincial ou territorial. Les différentes politiques scolaires provinciales ou territoriales peuvent donc s'opérationnaliser différemment selon les conseils, les centres de services ou les districts scolaires. Ici aussi, un élève pourrait ainsi être identifié comme doué dans un district scolaire, mais pas dans un autre (Klapp et Porath, 2000).

\section{Les élèves doués et talentueux}

Il n'y a pas de définition universellement acceptée de la douance. Les modèles théoriques de l'intelligence et de la douance ont évolué à travers le temps. D'abord dominées par des approches psychométriques (p. ex. : Cattell-Horn-Carroll) (Frey, 2018), où l'intelligence est conceptualisée comme une sorte de cartographie des facultés mentales et est typiquement évaluée à l'aide des tests de quotient intellectuel (QI), les définitions ont évolué vers des théories systémiques et des modèles développementaux (Sternberg, 2012). Actuellement, les définitions les plus souvent utilisées, selon McBee et Makel (2019) sont : (a) la définition d'une capacité cognitive élevée, (b) les définitions de critères multiples (dont le modèle à trois anneaux de Renzulli) et (c) la déclaration de principes de la National Association for Gifted Children (NAGC, 2019). On peut également ajouter à cette liste, le modèle intégrateur de développement du talent de Gagné (2009) ainsi que la théorie triarchique de Sternberg (2012).

\footnotetext{
${ }^{1}$ Le rapport Eurydice, cependant, indique que la définition de « doués et talentueux » est plus couramment utilisée dans toute l'Europe, bien qu'exprimée dans la langue du pays. Dans le même temps, il est nécessaire de souligner que dans le RoyaumeUni (et touchant la littérature), il y a une différence entre le terme « doué », qui indique généralement les élèves ayant des résultats académiques, et «talentueux » qui fait plutôt référence aux élèves avec des performances élevées dans les sports et les arts (White et al., 2003). En Europe francophone, le terme « haut potentiel » est également très utilisé (Clobert et Gauvrit, 2021). Au regard des politiques provinciales et territoriales en la matière, c'est l'appellation " doué et talentueux » qui sera utilisée dans cet article.
} 
Au niveau canadien, il n'y a pas de définition commune de la douance entre les provinces et territoires canadiens. Leur définition respective se trouve dans les différentes lois et politiques de scolarisation des élèves doués émanant des différents ministères et départements de l'éducation provinciaux et territoriaux. La plupart des politiques de scolarisation des élèves à besoins spécifiques au Canada ont connu depuis les 25 dernières années des changements reflétant une philosophie plus intégrative ou inclusive de l'éducation (Yewchuk, 2000; Leroux, 2000; Tremblay et Belley, 2017). Ainsi, l'offre de services répondant aux besoins des élèves doués semble avoir subi une évolution similaire aux élèves ayant des besoins spécifiques avec le passage de la ségrégation à l'inclusion (Smith, 2006). Cela a été particulièrement vrai en Amérique du Nord, où l'enseignement ordinaire a été longtemps considéré comme étant inapproprié pour les élèves doués (Montgomery, 2009). Cependant, comme Smith (2006) soutient également, bien que l'éducation inclusive insiste sur l'importance pour les systèmes scolaires d'accueillir tous les apprenants, dans les faits, l'inclusion s'est principalement orientée vers des élèves ayant des difficultés d'apprentissage (ou comportementales) et bien moins sur ceux qui démontrent de forts potentiels d'apprentissage.

$\mathrm{Au}$ niveau canadien, différentes enquêtes sur les politiques en matière de scolarisation des élèves doués ont été entreprises il y a plusieurs années (Goguen, 1993; Leroux, 2000; Yewchuk, 2000; Massé, 2000; Lupart et al., 2005). Cependant, différentes critiques ou limites peuvent être émises à propos de ces travaux. Premièrement, les politiques provinciales et territoriales de scolarisation des élèves à besoins spécifiques ont beaucoup changé depuis cette époque (p. ex. passage à l'éducation inclusive). Ensuite, ces écrits sont essentiellement descriptifs en cherchant à répertorier au mieux les politiques et dispositifs présents dans les provinces et territoires canadiens; sans dimension analytique. Leurs approches méthodologiques s'inspirent peu de celles développées en éducation comparée. Par ailleurs, on observe l'absence de cadre conceptuel (outre en ce qui concerne la douance). Enfin, les données portant sur les documents en langue française (essentiellement québécois) sont souvent lacunaires. Plus récemment, Kanevsky et Clelland (2013) ont réalisé une analyse des politiques scolaires relatives à la scolarisation des élèves doués et catégorisé 18 types de pratiques et dispositifs scolaires destinés aux élèves doués et talentueux dans toutes les provinces et territoires du Canada (voir annexe 2). Ces chercheurs remarquent également que là où elles ont été trouvées, les politiques sont bienveillantes envers ces élèves et souples dans les mesures pédagogiques. L'Alberta, la Colombie-Britannique, le Québec, le Nouveau-Brunswick et la Nouvelle-Écosse sont les provinces qui offrent un appui explicite à la scolarisation des élèves doués et aux mesures pédagogiques qui leur sont destinées. Toutefois, Kanevsky et Clelland (2013) observent que ces mesures pédagogiques sont également présentes, en tout ou en partie, dans l'ensemble des juridictions provinciales et territoriales pour ces élèves. Par ailleurs, la grande majorité des mesures pédagogiques identifiées à l'échelle du pays étaient orientées sur le contenu plutôt que sur le niveau scolaire.

\section{Méthodologie}

Dans ce cadre, réitérons l'objectif double de cette recherche: identifier les provinces préconisant une approche catégorielle face à la douance, pour en analyser les terminologies, définitions et domaines visés, tout en effectuant une analyse des mesures pédagogiques explicites ou implicites - offertes aux élèves doués dans l'ensemble des juridictions canadiennes. En effet, l'absence de prise en compte explicite de cette population par une province ou un territoire ne signifie pas qu'aucune mesure similaire ne puisse être prise pour des élèves, qu'ils soient identifiés comme doués ou non.

L'analyse des lois et des politiques scolaires entourant les services éducatifs offerts aux élèves ayant des besoins spécifiques a été effectuée à l'aide d'une typologie de textes scolaires officiels (Higgins et Barresi, 1979; Poirier et al., 1988; AuCoin et al., 2011; Tremblay, 2018). 
Les différents textes légaux ou administratifs encadrant l'inclusion scolaire peuvent être de différents types. Ainsi, ces textes ont été examinés à la lumière d'une typologie de trois grands types, allant de ceux du type 1 (Constitution, législations, conventions), aux politiques et directives ministérielles (type 2) et, enfin, aux guides spécifiquement rédigés pour décrire des mesures pédagogiques à utiliser pour ces élèves (type 3 ).

\subsection{Collecte des données}

Aux fins de la présente analyse, la collecte a permis de répertorier et de colliger l'ensemble des lois et des politiques gouvernementales spécifiques à chacun des provinces et des territoires canadiens, et ce, en allant directement les chercher sur les divers sites internet gouvernementaux. Chaque territoire $(n=3)$ et province canadiens $(n=10)$ a été inclus dans cette recherche de documents tant en langue française qu'anglaise. Plusieurs phases de collectes $(n=6)$ ont été nécessaires entre 2017 et 2021 pour s'assurer d'avoir un échantillon complet de données pertinentes (Gall et al., 2007; Kanevsky et Clelland, 2013). Trois types de documents de gouvernement disponibles en version imprimée ou en ligne ont été recherchés : (1) les lois et règlements qui régissent les services et programmes pour la scolarisation obligatoire; (2) les politiques portant sur la scolarisation des élèves doués et talentueux (3) les ressources documentaires créées pour les professionnels qui travaillent avec les élèves doués et leurs parents. Ce travail a permis de répertorier un total de 37 textes. Parmi ces textes se retrouvent au moins une législation et une politique par province et territoire (27) ainsi que quelques guides (10) s'adressant spécifiquement aux élèves doués et talentueux (tableau 1).

\section{Tableau 1 : Répertoire des lois, politiques gouvernementales et guides provinciaux et territoriaux}

\begin{tabular}{|c|c|c|}
\hline Provinces/Territoires & Date & Documents types 1 et 2 - Lois et politiques gouvernementales \\
\hline \multirow{7}{*}{ Alberta } & 2014 & Special Education Coding Criteria 2014-2015 \\
\hline & $2000-2013$ & School Act \\
\hline & 2000 & $\begin{array}{l}\text { Teaching Students Who Are Gifted and Talented - Programming for } \\
\text { Students with Special Needs }\end{array}$ \\
\hline & 2004 & Guide à l'intention des parents ayant un enfant doué et talentueux \\
\hline & 2008 & $\begin{array}{l}\text { Chapter 5: Students Who Are Gifted - Japanese Language and Culture } \\
\text { 9-year Guide to Implementation (4-6) }\end{array}$ \\
\hline & 2006 & $\begin{array}{l}\text { Chapter 11: Planning for Students Who Are Gifted. In Individualized } \\
\text { Program Planning (IPP): ECS to Grade } 12 .\end{array}$ \\
\hline & 2020 & Critères relatifs aux codes en adaptation scolaire $2020-21$. \\
\hline \multirow{3}{*}{$\begin{array}{l}\text { Colombie- } \\
\text { Britannique }\end{array}$} & 2013 & $\begin{array}{l}\text { Special Education Services: A Manual of Policies, Procedures and } \\
\text { Guidelines. }\end{array}$ \\
\hline & s.d. & Special Education: Gifted Education - A Resource Guide for Teachers \\
\hline & 1996-2016 & School Act \\
\hline \multirow{2}{*}{$\begin{array}{l}\text { Île-du-Prince- } \\
\text { Édouard }\end{array}$} & 2001 & Minister's Directive No. MD 2001-08. \\
\hline & $1993-2013$ & School Act \\
\hline \multirow{3}{*}{ Nouveau-Brunswick } & 2013 & Policy 322 Inclusive Education \\
\hline & $1997-2016$ & Loi sur l'éducation \\
\hline & 2007 & Gifted and Talented Students: A Resource Guide for Teachers \\
\hline \multirow[b]{2}{*}{ Manitoba } & $2006-2016$ & Loi sur les écoles publiques. \\
\hline & 2004 & $\begin{array}{l}\text { À l'appui des écoles favorisant l'inclusion : planification scolaire et } \\
\text { communication des renseignements : un cadre pour l'élaboration et la } \\
\text { mise en œuvre des plans scolaires et des rapports annuels. }\end{array}$ \\
\hline \multirow{3}{*}{$\begin{array}{c}\text { Terre-Neuve et } \\
\text { Labrador }\end{array}$} & $1997-2016$ & School Act \\
\hline & 2010 & $\begin{array}{l}\text { Provincial Strategy for the Inclusion of Persons with Disabilities in } \\
\text { Newfoundland and Labrador }\end{array}$ \\
\hline & 2013 & Teaching Student who are Gifted: A Handbook for Teachers \\
\hline
\end{tabular}




\begin{tabular}{|c|c|c|}
\hline \multirow{4}{*}{$\begin{array}{c}\text { Territoires du Nord- } \\
\text { Ouest }\end{array}$} & 2015 & Student Support Services - Gifted and Talented \\
\hline & 2016 & NWT Ministerial Directive on Inclusive Schooling \\
\hline & 2013 & Education Renewal and Innovation Framework : Directions for Change \\
\hline & 1995-2016 & Loi sur l'éducation \\
\hline \multirow{3}{*}{ Nouvelle-Écosse } & 2008 & Special Education Policy \\
\hline & 1995-2015 & Education Act. \\
\hline & 2011 & L'éducation des élèves doués et le développement des talents \\
\hline \multirow[b]{2}{*}{ Nunavut } & 2008 & Foundation for Inclusive Education Inuglugijaittuq in Nunavut Schools \\
\hline & 2008 & $\begin{array}{l}\text { Education Act: A Reference Guide for the Commission Scolaire } \\
\text { Francophone and School Principals }\end{array}$ \\
\hline \multirow{4}{*}{ Ontario } & 2009 & $\begin{array}{l}\text { Policy/Program Memorandum No 119, "Developing and Implementing } \\
\text { Equity and Inclusive Education Policies in Ontario Schools }\end{array}$ \\
\hline & 2008 & Successful Interventions for Gifted Learners in Ontario school \\
\hline & 1990-2016 & Education Act \\
\hline & 2017 & $\begin{array}{l}\text { Éducation de l'enfance en difficulté : guide pour les éducatrices et } \\
\text { éducateurs }\end{array}$ \\
\hline \multirow{4}{*}{ Québec } & 1988-2016 & Loi sur l'instruction publique. \\
\hline & 2000 & $\begin{array}{l}\text { Élèves handicapés ou élèves en difficulté d'adaptation ou } \\
\text { d'apprentissage (EHDAA) : définitions }\end{array}$ \\
\hline & 1999 & Politique de l'adaptation scolaire : une école adaptée à tous ses élèves. \\
\hline & 2020 & Agir pour favoriser la réussite éducative des élèves doués \\
\hline \multirow{6}{*}{ Saskatchewan } & 1995-2015 & The Education Act \\
\hline & 2006 & $\begin{array}{l}\text { Policy, Guidelines, and Procedures for Alternative Education Programs } \\
\text { Alternative Grade } 10,11 \text {, and } 12\end{array}$ \\
\hline & 2006 & Policy, Guidelines, and Procedures for Functional Integrated Programs \\
\hline & 2007 & The Disability Inclusion Policy Framework \\
\hline & 2007 & Policy and Procedures for Locally Modified Courses of Study \\
\hline & 2011 & $\begin{array}{l}\text { Actualizing a Needs-Based Model to Support Student Achievement: A } \\
\text { Journey to Transformation }\end{array}$ \\
\hline \multirow{2}{*}{ Yukon } & 2015 & Student Support Services Manual \\
\hline & $2002-2013$ & Education Act \\
\hline
\end{tabular}

\subsection{Méthodes d'analyse des données}

Suite à la recherche documentaire, l'analyse de contenu s'est faite dans une optique de catégorisation, permettant l'identification de thèmes. Ces derniers (terminologie, définition, domaine et mesures pédagogiques à l'intention des élèves doués et talentueux) visaient à faire ressortir les ressemblances et les différences entre les provinces et territoires canadiens quant à leurs approches face à la douance. Les étapes de la recherche documentaire ont été suivies (Fortin, 2010). Ainsi, pour chacun des thèmes à l'étude, la méthode d'analyse a fait appel au repérage d'indices contenus dans le corpus des textes associés répertoriés, ces indices ayant ensuite permis l'élaboration d'indicateurs déterminés autour desquels gravitent les idées présentées. Enfin, les données ont été contrôlées par des visites sur le terrain auprès des ministères concernés, des commissions scolaires et des écoles.

\subsection{Critères d'analyse des mesures pour élèves doués}

L'analyse des différentes mesures scolaires ou extrascolaires destinées aux élèves doués a été réalisée sur la base des travaux d'Eurydice (2006), de Kanevsky (2011) et de Kanevsky et Clelland (2013) en procédant à une traduction/adaptation en langue française des terminologies utilisées puis à une recatégorisation. Ainsi cinq catégories de mesures scolaires ou extrascolaires pour élèves doués sont utilisées: (1) le placement, (2) l'accélération, (3) l'enseignement différencié/individualisé, (4) les mesures extrascolaires, et (5) autres.

Les mesures de placement permettent d'orienter l'élève doué et talentueux vers des écoles ou des classes à caractéristiques spécifiques, et ce, tout en restant au même niveau 
scolaire. Cette catégorie comprend, d'une part, les mesures éducatives relatives à la fréquentation d'écoles ou de classes spéciales destinées aux élèves doués et talentueux et, d'autre part, la fréquentation de programmes spéciaux dont celui du Programme d'éducation internationale (PEI) ou Arts-études. Par ailleurs, dans le cadre de cet article, les mesures de placement incluent également les mesures d'enrichissement, ces dernières correspondant au fait que l'élève bénéficie formellement de cours enrichis (ex. cours de mathématiques avancées de $5^{\mathrm{e}}$ secondaire) d'un même niveau scolaire menant ainsi à l'obtention de crédits.

Les mesures d'accélération, elles, font référence aux différentes mesures éducatives permettant à l'élève doué et talentueux de bénéficier formellement, d'au moins, un cours, d'une classe, de niveau supérieur ${ }^{2}$. Ainsi, elles lui permettent de réaliser plus rapidement certains apprentissages, s'écartant par le fait même du cursus scolaire «traditionnel ». Par exemple, la catégorie des mesures d'accélération comprend le placement avancé, l'inscription double ou simultanée, les cours par correspondance (programme scolaire), l'accélération partielle et l'accélération par niveaux, l'entrée précoce dans différents ordres scolaires, la graduation précoce de même que le programme télescopique.

Les mesures d'enseignement différenciées/individualisées permettent à l'élève doué ou talentueux la possibilité d'évoluer à son rythme ou de suivre un programme particulier. La différenciation concerne tous les élèves alors que l'individualisation ne concerne qu'un élève. Toutes deux lui permettent entre autres de disposer de temps pour réaliser des apprentissages différents de ceux qui sont normalement prévus au programme et de développer ses aptitudes dans un domaine spécifique, que ce soit des aptitudes sportives, artistiques, créatives ou encore un intérêt particulier pour une matière. Il est à noter que ce type de mesures ne mène pas à l'obtention d'unités de cours supplémentaires, à la différence des mesures de placement. Sont compris dans cette catégorie : la progression continuelle des apprentissages, les séminaires et les projets spéciaux, sous-groupes de besoin, les centres d'apprentissage, le programme compacté (ou compression de programme), les études autonomes, les tâches par niveaux, le tutorat, le mentorat, les contrats de travail individuel, les programmes parascolaires ou extracurriculaires, le contrat d'apprentissage ainsi que les classes de développement de compétences réflexives.

Les mesures extrascolaires, quant à elles, sont des mesures éducatives réalisées en dehors du temps et de l'espace scolaire (contrairement aux activités parascolaires ou extracurriculaires qui prennent place à l'école). Elles permettent à l'élève de développer ses aptitudes dans un domaine spécifique (domaines sportif, artistique, etc.). Les clubs, les compétitions, les camps d'été spéciaux ou le bénévolat sont des exemples de mesures extrascolaires à l'intention des élèves doués ou talentueux. Les cours par correspondance (horsprogramme scolaire) sont également rangés dans cette catégorie.

Enfin, les mesures autres correspondent aux mesures ne pouvant pas être catégorisées selon les critères mentionnés ci-dessus. Cette catégorie regroupe ainsi les offres de bourses, les centres d'écoute, les réseaux de soutien de même que la présence d'un plan d'intervention chez l'élève doué et talentueux, pour n'en nommer que quelques exemples.

\section{Résultats}

La revue des législations (type 1) et des politiques (type 2) spécifiques à chacun des provinces et des territoires canadiens a mis en évidence que sur l'ensemble des 13 juridictions, seulement quatre provinces (Alberta, Colombie-Britannique, Ontario et Terre-Neuve-et-Labrador) reconnaissent légalement une catégorie d'élèves doués et talentueux. Ces provinces sont

\footnotetext{
2 Selon Colangelo et al. (2004), les 18 types d'accélération potentiellement mis à la disposition des élèves doués sont répartis en deux catégories principales : les programmes accélérés en fonction du niveau scolaire (ex. : réduisent le nombre d'années passées en primaire) et les programmes accélérés en fonction des matières, qui offrent un contenu plus avancé que les programmes traditionnels.
} 
considérées catégorielles puisque leur politique respective reconnaît et définit une catégorie spécifique pour ces élèves. Pour ce qui est de l'Ontario, bien qu'elle distingue la catégorie des élèves doués et talentueux, aucun document concernant l'identification de cette population n'est disponible. Ceci peut s'expliquer par le fait que les conseils scolaires ontariens sont responsables de l'élaboration des critères et des domaines relatifs à cette catégorie.

Toutefois, sans leur attribuer une catégorie particulière, les élèves doués et talentueux apparaissent dans les textes de quatre autres juridictions : Saskatchewan, la Nouvelle-Écosse, le Nouveau-Brunswick et les Territoires du Nord-Ouest. Premièrement, la Saskatchewan propose un article de loi (type 1) concernant les élèves doués et talentueux sans pour autant bénéficier de politiques ou de guides spécifiques à leur attention : "Lorsque les programmes d'enseignement ordinaires de l'école sont considérés par la commission scolaire ou le conseil scolaire comme étant insuffisants pour répondre aux besoins éducatifs de certains élèves ayant des aptitudes supérieures ou un talent exceptionnel, la commission scolaire ou le conseil scolaire peut prévoir la possibilité que l'élève bénéficie d'un programme spécial, s'il juge que c'est approprié et réalisable » (Saskatchewan Government, 1995, article 187).

Similairement, sans posséder d'article de loi ni de politique concernant les élèves doués et talentueux, la Nouvelle-Écosse, le Nouveau-Brunswick et le Québec ont respectivement produit un guide à l'intention des enseignants (type 3) dans lequel les provinces définissent la douance. Dans la mesure où il n'existe pas de catégorie pour les élèves doués et talentueux dans les politiques de ces trois juridictions, aucun financement spécifique ne leur est offert; l'élaboration de ces guides vise plutôt à apporter une aide aux enseignants afin de favoriser l'intégration des élèves doués en classe ordinaire. Il est d'ailleurs à noter que le document québécois souligne à plusieurs reprises que les mesures suggérées ne sont pas limitées aux élèves doués. Elle ne manque pas de préciser à plusieurs reprises que « la douance ne doit pas être considérée comme un diagnostic " (Ministère de l'Éducation et de l'Enseignement supérieur, 2020, p.6). Quant à eux, les Territoires-du-Nord-Ouest ont recours à l'appellation " élèves en besoin d'enrichissement ", laquelle apparaît dans la plus récente politique sur l'éducation inclusive (Northwest Territories Department of Education, Culture and Employment, 2016, p. 8). Or, aucune définition de cette population d'élèves n'est donnée.

À la lumière de ces précisions, la Saskatchewan, la Nouvelle-Écosse, le NouveauBrunswick, le Québec et les Territoires-du-Nord-Ouest ne seront pas considérés comme des juridictions reconnaissant officiellement cette catégorie dans le cadre de cet article. Compte tenu du fait que la prochaine section a pour objectif d'analyser des définitions de la douance, il sera ainsi question uniquement des quatre provinces qui en proposent explicitement et officiellement une (Alberta, Colombie-Britannique, Ontario et Terre-Neuve-et-Labrador).

\subsection{Définition de la douance}

Tel que vu plus haut, quatre provinces reconnaissent explicitement cette catégorie d'élèves (Alberta, Colombie-Britannique, Ontario et Terre-Neuve-et-Labrador), lui assignant un plan d'intervention. Il importe de rappeler que cette reconnaissance comme élèves à besoins spécifiques signifie également la possibilité d'adapter et même modifier (augmenter) les objectifs d'apprentissage, contrairement aux pratiques de modifications habituellement utilisées qui consistent à diminuer les exigences scolaires pour des élèves ayant des limitations importantes.

\subsubsection{Terminologie}

Parmi les différentes terminologies utilisées par les quatre provinces catégorielles pour désigner les élèves doués et talentueux, il est possible de mettre en évidence l'emploi plus fréquent de deux termes distincts : « doués » et « talentueux ». Tandis que certaines juridictions semblent utiliser une seule terminologie à des fins d'uniformisation, d'autres les utilisent en 
combinaison pour faire état de la même population d'élèves à besoins spécifiques. Par exemple, les quatre provinces (Alberta, Colombie-Britannique, Ontario et Terre-Neuve-et-Labrador) réfèrent au terme "doué » pour désigner les élèves doués et talentueux. Ajoutons que pour l'Alberta et l'Ontario, une variation dans le terme est parfois employée : tantôt sera utilisé le terme « doué », tantôt « surdoué » (Ministère de l'Éducation de l'Ontario, 2017). Cependant, l'Ontario utilise les deux termes sans distinction, alors que l'Alberta précise que l'appellation « surdoué » réfère aux élèves qui « ont un QI allant de 145 à 159 ou plus » (Alberta Education, 2002, p. 92).

Par ailleurs, les provinces de Terre-Neuve-et-Labrador et de l'Alberta sont les seules à joindre le terme talentueux à leurs définitions (Alberta Learning, 2004, p. 14; Newfoundland and Labrador Department of Education and Early Childhood, 2015). Or, alors que la première n'effectue aucune distinction entre les deux termes, l'Alberta réserve le terme " doué » aux domaines intellectuel ou académique tandis que le terme «talentueux » est réservé aux domaines artistique et sportif (Alberta Learning, 2004, p. 14). Il est à noter que le terme talentueux n'est jamais utilisé seul.

\subsubsection{Les multiples formes de la douance}

L'Alberta, dans sa définition de la douance, met en évidence le large éventail de comportements et d'aptitudes associés à cette catégorie d'élèves en indiquant que " le don est un potentiel exceptionnel et /ou des performances dans un large éventail de compétence ... » (Alberta Learning, 2000, p. 27; Alberta Education, 2014, p. 5). À l'inverse, les définitions offertes par la Colombie-Britannique, l'Ontario et Terre-Neuve-et-Labrador ne permettent pas de dégager le caractère diversifié de la douance de façon aussi explicite.

\subsubsection{Doublement exceptionnel}

Un autre attribut, ayant pu être dégagé des définitions de la douance de certaines provinces, concerne la présence d'un autre caractère exceptionnel. Deux provinces font ainsi état de cette spécificité dans leur définition. C'est le cas de Terre-Neuve-et-Labrador dont la définition indique que la douance peut « coexister avec un autre caractère exceptionnel » (Newfoundland and Labrador Department of Education and Early Childhood, 2015, p.1). Le ministère de l'Éducation de la Colombie-Britannique, quant à lui, indique qu'il peut également y avoir « des déficiences qui accompagnement [la douance et qu'on ne peut] s'attendre à avoir des atouts dans tous les domaines du fonctionnement intellectuel »(British Columbia Ministry of Education, 2013, p. 53).

\subsubsection{Besoins différenciés}

Les quatre provinces (Alberta, Colombie-Britannique, Ontario et Terre-Neuve-et-Labrador) considèrent les élèves doués et talentueux comme étant une catégorie d'élèves à besoins spécifiques. Or, seulement deux de ces mêmes provinces ajoutent à leur définition respective de la douance la nécessité de fournir à ces élèves des programmes différenciés adaptés à leurs besoins. C'est ainsi le cas de l'Ontario dont la définition propose «des expériences d'apprentissages différenciés d'une profondeur et d'étendue au-delà de celles normalement prévues dans le programme scolaire régulier ... » (Ministère de l'Éducation de l'Ontario, 2017, A20) et de l'Alberta pour qui les élèves doués et talentueux « requièrent des conditions et des programmes différenciés pour atteindre leur plein potentiel » (Alberta Learning, 2004, p.14). Les deux autres juridictions (Colombie-Britannique et Terre-Neuve) sont muettes sur le sujet malgré l'existence de plusieurs mesures pédagogiques proposées aux élèves doués et talentueux (tableau 3).

\subsubsection{Domaines de la douance}


La douance est un caractère exceptionnel pouvant se manifester à travers plusieurs domaines distincts. Dans le cadre de cette analyse seront considérées quatre catégories de domaines : (1) intellectuel, (2) social, (3) artistique et (4) psychomoteur. Ces catégories sont celles utilisées dans les textes de ces quatre provinces. Le domaine intellectuel réfère aux aptitudes cognitives tant à ce qui a trait au cadre scolaire (aptitudes linguistiques et mathématiques) qu'aux activités plus transversales telles que le raisonnement inductif et déductif, les résolutions de problèmes ainsi que la visualisation et la gestion de l'espace. Le domaine social englobe les compétences sociales; sont comprises dans ce domaine les capacités de «leadership », de persuasion, de compréhension, d'empathie et la gestion des émotions. Le troisième domaine, celui artistique, comprend les aptitudes créatives de l'ensemble des champs d'expression artistiques. Par exemple, la danse, la musique, les arts plastiques et la pensée créatrice appartiennent au domaine artistique. Enfin, le domaine psychomoteur est davantage orienté vers les aptitudes motrices utilisées dans un cadre sportif, de danse ou dans le but de poser des actes techniques précis (ex. arts du cirque).

D'emblée, tous les élèves vont présenter des habiletés appartenant aux quatre différentes catégories de domaines. Or, les élèves doués et talentueux vont, quant à eux, manifester un degré supérieur de réalisation dans l'un ou l'autre de ces domaines; il ne s'agit donc pas d'une dichotomisation, mais plutôt d'une question de degré. C'est en ce sens que l'utilisation de qualificatifs est importante lors de l'énumération des critères de réalisation. L'utilisation de ces qualificatifs rend toutefois les évaluations de la douance possiblement plus subjectives.

Tableau 2 : Domaines de la douance par provinces

\begin{tabular}{|c|c|c|c|c|}
\hline Domaines & ALB & CB & ONT & TNL \\
\hline \hline Intellectuel & $\mathrm{X}$ & $\mathrm{X}$ & & $\mathrm{X}$ \\
\hline Social & $\mathrm{X}$ & & & $\mathrm{X}$ \\
\hline Artistique & $\mathrm{X}$ & $\mathrm{X}$ & & $\mathrm{X}$ \\
\hline Psychomoteur & $\mathrm{X}$ & & & $\mathrm{X}$ \\
\hline
\end{tabular}

Le tableau 2 montre que les provinces s'étant doté d'une définition de la douance en abordent généralement plusieurs, sinon tous, les domaines. Cette tendance est ainsi présente pour quatre provinces, à l'exception de l'Ontario qui ne fait d'ailleurs référence à aucun domaine dans la définition qu'elle propose de la douance. Rappelons qu'en Ontario, il est de la responsabilité des conseils scolaires de déterminer les critères et les indicateurs permettant l'identification des élèves doués et talentueux. Malgré le fait que cette province ne précise pas explicitement que la douance peut se manifester à travers le domaine intellectuel, l'Ontario semble néanmoins mettre cet aspect en évidence, que ce soit dans la définition même d'un élève présentant ce caractère exceptionnel que dans l'élaboration des programmes d'apprentissage leur étant destinés : «Enfant d'un niveau mental très supérieur à la moyenne, qui a besoin de programmes d'apprentissage beaucoup plus élaborés que les programmes réguliers et mieux adaptés à ses facultés intellectuelles » (Ministère de l'Éducation de l'Ontario, 2017, A20).

En ce qui est de la Colombie-Britannique, il importe de nuancer le fait que malgré l'énumération explicite des domaines « intellectuel» et « artistique » uniquement, un élève peut satisfaire les critères d'éligibilité à la douance s'il démontre de grandes habiletés associées à un autre domaine spécifique non déterminé par la province (British Columbia Ministry of Education, 2010, p. 13).

\subsection{Mesures scolaires et extrascolaires}

Bien que la majorité des provinces et territoires canadiens soient dépourvus de définition officielle de la douance ou que les élèves doués et talentueux ne soient pas reconnus comme catégorie d'élèves à besoins spécifiques, il peut exister néanmoins des mesures pédagogiques 
similaires à celles proposées par les quatre provinces pour lesquelles on retrouve une définition. Prosaïquement, il est possible de faire un saut d'année dans tous les systèmes scolaires canadiens sans pour autant être reconnu comme doué.

Sur l'ensemble des mesures éducatives indiquées (tableau 3), il semble que cinq d'entre elles soient davantage partagées par les provinces et les territoires canadiens, soit (1) les bourses françaises/anglaises, (2) la différenciation pédagogique, (3) le PEI, (4) les cours par correspondance et (5) les mesures d'accélération toutes formes confondues : l'accélération totale, l'accélération partielle et le placement avancé. Les bourses d'excellence aux élèves, le PEI ainsi que les cours par correspondance constituent les trois mesures à être proposées dans toutes les juridictions. Toutefois, le PEI et les cours par correspondance ont été explicitement répertoriés dans les guides pédagogiques de respectivement huit et cinq juridictions. D'ailleurs, les quatre provinces ayant une définition de la douance en font mention explicitement, à l'exception de la Colombie-Britannique pour les cours par correspondance. Pour les autres provinces et territoires, l'information relative à l'existence de ces deux mesures n'apparait donc pas directement dans les guides s'inscrivant dans le cadre de cette analyse. Par ailleurs, quant au programme du PEI, soulignons qu'il semble s'agir de la seule mesure de placement offerte par la Nouvelle-Écosse avec le groupement par habiletés.

Les mesures de différenciation pédagogique sont partagées par 11 provinces et territoires sur 13 (sauf Territoires-du-Nord-Ouest et Nouvelle-Écosse). On peut toutefois aisément supposer que malgré l'absence d'explicitation à l'intérieur des politiques et des guides des juridictions des Territoires du Nord-Ouest et de la Nouvelle-Écosse, la différenciation pédagogique, par sa nature, est d'emblée une mesure éducative qui peut être effectuée auprès des élèves à besoins spécifiques, et, par le fait même, auprès des élèves doués et talentueux (Northwest Territories Department of Education, Culture and Employment, 2016; Nova Scotia Government, 2008). De plus, il importe de considérer le caractère large associé à la mesure de différenciation pédagogique englobant ainsi une multitude de pratiques. Par ailleurs, la Nouvelle-Écosse et l'Ontario représentent les provinces pour lesquelles il semble exister le moins de mesures de différenciation à l'égard des élèves doués et talentueux. À l'inverse, la province de Terre-Neuve-et-Labrador apparaît comme celle offrant le plus de mesures associées à cette catégorie. D'ailleurs, deux mesures de différenciation semblent exclusives à cette province : l'étude de célébrités et le contrat d'apprentissage.

Par ailleurs, si une province peut organiser des écoles ou classes spéciales à l'intention des élèves doués, ces classes ou écoles restent rares ${ }^{3}$. Par exemple, pour les deux provinces catégorielles que sont l'Alberta et l'Ontario, seulement quelques écoles se spécialisent dans la scolarisation des élèves doués. Le Québec, récemment, a reconnu explicitement qu' " une classe spéciale à temps plein ou à temps partiel peut parfois répondre aux besoins de ceux qui vivent des difficultés. Le Ministère soutient financièrement une variété de regroupements d'élèves » (Ministère de l'Éducation et de l'Enseignement supérieur, 2020, p.18). Le groupement par habiletés est toutefois possible dans sept provinces et territoires. Enfin, Terre-Neuve-etLabrador est encore une fois celle présentant une plus grande variété de mesures de placement; plusieurs d'entre elles semblant même exclusives à cette province. En effet, plus de la moitié des mesures de placement offertes par cette province n'apparaît chez aucune autre province catégorielle : les cours externes, le placement alternatif, les mini-cours et les cours de courte durée.

Les mesures d'accélération (selon les formes) sont partagées universellement par les différents provinces et territoires canadiens. Il importe de préciser qu'alors que la majorité des juridictions font référence explicitement aux mesures d'accélération totale, la Colombie-

\footnotetext{
${ }^{3}$ Des provinces ne reconnaissant pas cette catégorie d'élèves peuvent cependant avoir des établissements scolaires publics ou privés réservés en tout ou en partie à cette population (sur la base d'examen psychologique et/ou scolaire). Ainsi, au Québec existent des classes et des écoles sur la base d'un examen scolaire d'admission.
} 
Britannique et la Nouvelle-Écosse, pour leur part, considèrent plutôt le terme « placement avancé » pour renvoyer à la même réalité (British Columbia Ministry of Education, 2013; Nova Scotia Department of Education, 2011). À la lumière de cette nuance, on constate que différentes mesures d'accélération totale sont partagées par 10 provinces et territoires canadiens.

Enfin, parmi les cinq catégories de mesures, il semble que les mesures extrascolaires soient celles pour lesquelles il existe une diversité moindre, et ce, pour chaque province. Les programmes extrascolaires et les cours par correspondance paraissent les plus fréquents. Alors que les programmes extrascolaires sont offerts par quatre juridictions canadiennes, les cours par correspondance, eux, sont offerts sur tout le territoire canadien. Par ailleurs, Terre-Neuveet-Labrador connaît encore une fois le plus de mesures extrascolaires pour les élèves doués et talentueux, avec quatre mini-cours, centres d'apprentissage, programme spécial et cours par correspondance (hors-programme scolaire), en comparaison aux provinces pourvues, elles aussi, d'une définition de la douance qui n'offrent comme mesures extrascolaires que les cours par correspondance (hors-programme scolaire).

\section{Discussion}

Cet article avait pour objectif premier d'identifier les provinces qui ont une catégorie d'élèves doués pour y analyser les terminologies employées, les définitions utilisées et les domaines dans lesquels cette catégorie est susceptible de s'inscrire. Tout d'abord, on remarque que quatre provinces reconnaissent explicitement cette catégorie d'élèves (Alberta, ColombieBritannique, Ontario, Terre-Neuve-et-Labrador). La Nouvelle-Écosse, le Québec et le Nouveau-Brunswick ont toutefois produit un guide à l'intention des enseignants (type 3) malgré que les élèves doués et talentueux ne soient pas explicitement catégorisés dans les politiques (type 2) ou les législations (type 1). Cette situation met en exergue le fait que la noncatégorisation est administrative et d'ordre scolaire; elle ne conteste pas l'existence de ces catégories à d'autres fins. Ensuite, il semble y avoir un consensus sur le concept de douance et les termes « doué » et « talentueux » (gifted and talented, en anglais). Il y a une similitude avec les termes retrouvés au Royaume-Uni (European Agency for Development in Special Needs Education, 2009; Eurydice, 2006) et aux États-Unis (Davis \& Rimm, 1989; Heller et al., 2000). En Alberta, cette reconnaissance s'associe aussi à un financement individualisé en plus de leur permettre d'accéder à certains dispositifs réservés pour cette catégorie d'élèves (classe ou école spéciale).

Pour ce qui est des domaines de la douance, la sphère intellectuelle prédomine manifestement dans les définitions des quatre provinces identifiées comme catégorielles. Ce résultat confirme la conception selon laquelle il s'agit encore aujourd'hui d'une catégorie d'élèves prenant majoritairement appui sur des critères d'ordre intellectuel. Or, pour identifier les élèves doués et talentueux, les provinces semblent manifester la volonté de s'éloigner graduellement des approches psychométriques pour se tourner vers d'autres (théories systémiques et des modèles développementaux) (Frey, 2018; Newfoundland and Labrador Department of Education, 2013).

Le second objectif était d'analyser les mesures mises en œuvre pour ces élèves (explicitement ou implicitement) dans l'ensemble des provinces et territoires canadiens. Une première distinction peut s'établir entre les provinces et les territoires canadiens: les Territoires-du-Nord-Ouest, le Yukon et le Nunavut semblent offrir significativement moins de mesures éducatives à l'intention des élèves doués et talentueux que l'ensemble des autres juridictions. À cet effet, on peut supposer l'existence d'une relation entre, d'une part, les quelques mesures éducatives explicitement mentionnées dans les guides respectifs de ces territoires et, d'autre part, la faible population de chacun d'eux qui, malgré le fait que la proportion d'enfants doués soit sensiblement la même que dans les autres provinces, fait que 
leur effectif est en lui-même très petit. D'ailleurs, considérant le fait que les trois territoires canadiens sont dépourvus d'une définition de la douance, il semble cohérent que très peu de mesures éducatives existent pour les élèves doués et talentueux. En effet, on observe que les provinces catégorielles qui reconnaissent la douance ont plus de mesures, celles-ci sont plus structurées et organisées pour les élèves doués et qu'elles sont souvent plus aisément accessibles (ex. : guide pour enseignants). Ces résultats font donc écho aux conclusions de Kanevsky et Clelland (2013) soulignant un alignement entre la préconisation ou non d'une approche catégorielle au regard de la douance et le type et le nombre de mesures proposées à ces élèves. Les provinces ayant une définition de la douance apparaissent comme étant celles offrant une plus grande diversité de mesures éducatives, et, à l'inverse, les provinces non catégorielles sont généralement plus pauvres en mesures explicites, comme observé également par Kanevsky et Clelland (2013). Si le Québec, le Nouveau-Brunswick et la Nouvelle-Écosse présentent une variété de mesures éducatives à l'attention des élèves doués et talentueux, c'est que les provinces disposent d'un guide élaboré spécifiquement pour ces élèves à besoins spécifiques (New Brunswick, 2007). Or, puisqu'il n'existe aucune définition officielle des élèves doués et talentueux pour ces trois provinces, on peut penser que les mesures éducatives qu'elles proposent sont d'abord destinées à tous les élèves, mais que ceux doués et talentueux peuvent en bénéficier. Le Québec argumente dans le même sens avec son guide, en insistant fréquemment sur le fait que les mesures proposées pour les élèves doués peuvent répondre aux besoins d'un plus grand éventail d'élèves (Ministère de l'Éducation et de l'Enseignement supérieur, 2020).

Cependant, on ne voit pas de véritable lien entre les mesures et les différents domaines de la douance outre en ce qui concerne le domaine intellectuel (et artistique dans une moindre mesure). On voit ici une tension entre une définition de la douance et ses domaines, qui peuvent être plus larges que les programmes scolaires (p. ex. : social, artistique, psychomoteur). Il est utile de noter que les pratiques de différenciation pédagogique présentées sont susceptibles de convenir à la plupart des élèves. À l'exception de classes spéciales, les autres mesures de placement (ex. PEI, cours de l'enseignement supérieur, cours enrichi, etc.) sont fréquentées majoritairement par des élèves qui ne sont pas identifiés comme doués ou talentueux. Par ailleurs, les pratiques d'accélération comme l'accélération totale (saut de classe, entrée précoce dans un ordre d'enseignement, etc.) ou l'obtention de crédits par la réussite d'un examen peuvent convenir à des élèves qui ne sont pas nécessairement diagnostiqués comme doués ou talentueux. Toutefois, des procédures spécifiques peuvent être demandées pour bénéficier de ces mesures (ex. Québec). En ce sens, on retrouve la variabilité et la flexibilité des mesures destinées aux élèves doués observées chez Kanevsky et Clelland (2013). On observe également que malgré l'absence de reconnaissance explicite de la douance, l'accès à des opportunités d'apprentissage adaptées à leurs besoins, qui est identifié comme prioritaire pour le développement des élèves doués, est mis en œuvre par l'ensemble des provinces et territoires canadiens, bien que cela semble s'actualiser à différents niveaux selon les zones scolaires. Ainsi, afin de répondre aux besoins des élèves doués, une interprétation et une implantation flexible et délibérée, d'une part, et la mise en œuvre de politiques bienveillantes envers ces élèves, d'autre part, prennent de plus en plus d'importance à mesure que les philosophies et les pratiques en matière de scolarisation des élèves à besoins spécifiques deviennent moins catégorielles et plus inclusives (Kanevsky et Clelland, 2013).

Ainsi, relativement peu de mesures sont réservées exclusivement à cette population tant en théorie qu'en pratique. Des pratiques comme le programme compacté et l'inscription double ou simultanée paraissent toutefois plus destinées explicitement à ces élèves. Par ailleurs, la présence de catégorie ne garantit pas nécessairement que l'ensemble de la gamme de services soit disponible pour tous les élèves doués ou talentueux. Ainsi, une éducation inclusive, s'affranchissant des catégories, peut se centrer sur les besoins de l'élève, sur leur nature. La 
dispersion géographique de certaines populations ou leur faible densité peut conduire à proposer une moins grande diversité de services. La présence de guide pédagogique ne garantit quant à elle pas non plus que ceux-ci sont connus ou utilisés. On sait par ailleurs que les contenus concernant la douance dans la formation des enseignants sont réduits, sinon nuls (Leroux, 2000, Massé, 2000, Bergeron et St-Vincent, 2011). De plus, il existe peu de statistiques sur le nombre d'enfants doués et talentueux dans les écoles spéciales au Canada.

Enfin, les résultats montrent, bien entendu, un tableau contrasté entre provinces catégorielles ou non catégorielles, mais également une commune évolution, influencée par l'inclusion scolaire, vers des approches centrées sur les besoins propres de l'élève plutôt que sur sa catégorie. De plus, l'utilisation de pratiques probantes et universelles (efficaces pour la plupart des élèves) conduit à revoir les dispositifs ségrégatifs ou réservés à ces élèves. Par ailleurs, les capacités de plus en plus grandes des écoles canadiennes à scolariser les élèves à besoins spécifiques semblent rendre de moins en moins pertinents ce type de dispositifs.

\section{Références}

ABC Ontario. (2008). Successful interventions for gifted learners in Ontario schools. Toronto: Ministry of Education.

Alberta Education. (2002). Enseigner aux élèves doués et talentueux. Edmonton : Alberta Government.

Alberta Education. (2008). Chapter 5: Students who are gifted. Dans Japanese language and culture $9 Y$ guide to implementation (4-6). (pp. 93-106), Alberta Government.

Alberta Education. (2014). Special education coding criteria 2014-2015. Edmonton: Alberta Government

Alberta Education. (2020). Critères relatifs aux codes en adaptation scolaire 2020-21. Edmonton: Government of Alberta. Retrieved from https://open.alberta.ca/publications/1911-494x.

Alberta Government. (2000-2013). School act. Chapter S-3. Edmonton: Alberta Queen's Printer.

Alberta Learning. (2000). Teaching students who are gifted and talented. Dans Programming for Students with Special Needs (p. 1-367), Edmonton: Special Education Branch.

Alberta Learning. (2004). Le voyage : guide à l'intention des parents ayant un enfant doué et talentueux. Edmonton: Alberta Government.

Alberta Learning. (2006). Chapter 11: Planning for students who are gifted. Dans Individualized program planning (IPP: $\quad$ ECS to grade 12. (pp. 2-53). Edmonton: Alberta Education. https://education.alberta.ca/media/525558/ipp92.pdf

AuCoin, A., Goguen, L. et Vienneau, R. (2011). Pas plus spécial que nécessaire : analyse des politiques scolaires de la Nouvelle-Écosse à l'égard de l'inclusion scolaire des élèves avec handicaps. Éducation et francophonie, 39(2), 23-49.

Bergeron, G. et St-Vincent, L.-A. (2011). L’intégration scolaire au Québec : regard exploratoire sur les défis de la formation à l'enseignement au primaire et préscolaire. Éducation et francophonie. 39(2), 272-295.

Borland, J. (2005). Gifted education without gifted children: The case for no conception of giftedness. Dans R. J. Sternberg et J. E. Davidson (dir.), Conceptions of giftedness ( $2^{\mathrm{e}}$ éd., pp. 1-19). New York, NY: Cambridge University Press.

British Columbia Government. (1996). School act.

British Columbia Ministry of Education. (s.d.). Special education: Gifted education - A resource guide for teachers. Edmonton: BC Government. Retrieved from http://www.bced.gov.bc.ca/specialed/gifted/

British Columbia Ministry of Education. (2010). Special education services: Categories checklists - 2010. BC Government.

British Columbia Ministry of Education. (2013). Special education services: A manual of policies, procedures and guidelines. Victoria: BC Government.

Clobert, N. et Gauvrit, N. (2021). Psychologie du haut potentiel : comprendre identifier accompagner. Bruxelles : De Boeck Supérieur.

Colangelo, N., Assouline, S. G. et Gross, M. U. (2004). A nation deceived: How schools hold back America's brightest Students. The Templeton national report on acceleration. Vol. 2. Connie Belin \& Jacqueline N. Blank International Center for Gifted Education and Talent Development (NJ1).

Davis, G. A. et Rimm, S. B. (1989). Education of the gifted and talented (2e éd.). Englewood Cliffs, NJ: PrenticeHall.

European Agency for Development in Special Needs Education. (2009). Gifted learners: A survey of educational policy and provision. Bruxelles : European Agency for Development in Special Needs Education. 
Eurydice. (2006). Mesures éducatives spécifiques pour la promotion de tous les talents en Europe : document de travail. Bruxelles : Direction générale de l'éducation et de la culture.

Fortin, M.-F. (2010). Fondements et étapes du processus de recherche : méthodes quantitatives et qualitatives. $2^{\mathrm{e}}$ édition. Chenelière Éducation : Montréal.

Frey, B. (2018). The SAGE encyclopedia of educational research, measurement, and evaluation (vols. 1-4). Thousand Oaks, CA: SAGE Publications, Inc.

Gagné, F. (2009). Building gifts into talents: Detailed overview of the DMGT 2.0. Dans B. MacFarlane et T. Stambaugh (dir.), Leading change in gifted education: The festschrift of Dr. Joyce VanTassel-Baska (pp.61-80). Waco, TX: Prufrock Press.

Gall, M. D., Gall, J. P. et Borg, W. R. (2007). Educational research: An introduction. Boston: Pearson Education.

Goguen, L. J. (1993). Right to education for the gifted in Canada. Dans K. A. Heller, F. J. Mönks et Passow, A. H. (dir.), International handbook of research and development of giftedness and talent (p. 771-777). Oxford, UK: Pergamon.

Gouvernement du Manitoba. (2006-2016). Loi sur les écoles publiques. Winnipeg : Manitoba Government.

Gouvernement du Nouveau-Brunswick. (1997-2016). Loi sur l'éducation. Fredericton : NB Government.

Gouvernement du Québec. (1988-2016). Loi sur l'instruction publique. Chapitre I-13.3, legisquebec.gouv.qc.ca/fr/showdoc/cs/I-13.3

Government of Ontario. (1990-2016). Education Act. Toronto: Government of Ontario.

Government of Yukon. (2002-2013). Education Act. Whitehorse: Yukon Government.

Government of Yukon. (2015). Student support services manual. Whitehorse: Yukon Government.

Heller, K. A., Mönks, F. J., Subotnik, R. et Sternberg, R. J. (dir.). (2000). International handbook of giftedness and talent. Elsevier.

Higgins, S. et Barresi, J. (1979). The changing forces of public policy. Exceptional Children, 45(4), $270-277$.

Kanevsky, L. (2011). A survey of educational acceleration practices in Canada. Canadian Journal of Education, 34(3), 153-180.

Kanevsky, L. et Clelland, D. (2013). Accelerating gifted students in Canada: Policies and possibilities. Canadian Journal of Education, 36(3), p. 229-271.

Klapp, J. et Porath, M. (2000). Past, present and future of gifted education in British Columbia. AGATE-Journal of the Gifted and Talented Education Council of the Alberta Teachers'Association, 14(2), 26-35.

Leroux, J. A. (2000). A study of education for high ability students in Canada: Policy, programs and student needs. Dans K. A. Heller, F. J. Mönks et R. Subotnik (dir.), International handbook of giftedness and talent ( $\mathrm{p}$. 695-702), Oxford: Elsevier Science Ltd.

Lupart, J. L., Pyryt, M. C., Watson, S. L. et Pierce, K. (2005). Gifted education and counselling in Canada. International Journal for the Advancement of Counselling, 27(2), 173-190.

Manitoba Education. (2004). Á l'appui des écoles favorisant l'inclusion : planification scolaire et communication des renseignements : un cadre pour l'élaboration et la mise en auvre des plans scolaires et des rapports annuels. Winnipeg : Manitoba Government.

Massé, L. (2000). Gifted education in Quebec: A short past, a few appearances, and almost no future! AGATE Journal of the Gifted and Talented Education Council of the Alberta Teachers' Association, 14(2), 6071.

McBee, M. T. et Makel, M. C. (2019). The quantitative implications of definitions of giftedness. AERA Open, 5(1), 2332858419831007.

Minister of Education and Lifelong Learning. (2001). Minister's Directive No. MD 2001-08. Charlottetown: Prince Edward Island Government.

Ministère de l'Éducation de l'Ontario. (2009). Policy/program memorandum No 119, "Developing and implementing equity and inclusive education policies in Ontario schools. Toronto: Ministry of Education.

Ministère de l'Éducation de l'Ontario. (2017). Éducation de l'enfance en difficulté : guide pour les éducatrices et éducateurs. Toronto : Fonction publique de l'Ontario.

Ministère de l'Éducation et de l'Enseignement supérieur. (2020). Agir pour favoriser la réussite éducative des élèves doués. http://www.education.gouv.qc.ca/fileadmin/site_web/documents/education/adaptationscolaire-services-comp/Reussite-educative-eleves-doues.pdf

Ministère de l'Éducation, du Loisir et du Sport. (1999). Politique de l'adaptation scolaire : une école adaptée à tous ses élèves. Québec.

Ministère de l'Éducation, du Loisir et du Sport. (2000). Élèves handicapés ou élèves en difficulté d'adaptation ou d'apprentissage (EHDAA) : définitions. Gouvernement du Québec.

Montgomery, D. (2009). Effective teaching and learning to combat underachievement. Dans D. Montgomery (dir.) Able, gifted and talented underachievers pp. 111-154. https://doi.org/10.1002/9780470741252.ch5

National Association for Gifted Children. (2019). 2019 Pre-K-Grade 12 gifted programming standards. Washington, DC. 
Newfoundland and Labrador Government. (1997-2016). School Act. St-Johns: Government of Newfoundland and Labrador.

Newfoundland and Labrador Department of Education. (2010). Provincial strategy for the inclusion of persons with disabilities in Newfoundland and Labrador. St-Johns: Government of Newfoundland and Labrador

Newfoundland and Labrador Department of Education. (2013). Teaching student who are gifted: A handbook for teachers. St-Johns: Government of Newfoundland and Labrador.

Newfoundland and Labrador Department of Education and Early Childhood. (2015). Gifted and talented. Government of Newfoundland and Labrador. https://www.gov.nl.ca/education/k12/studentsupportservices/gifted/

Northwest Territories Department of Education, Culture and Employment. (2013), Education renewal and innovation framework: Directions for change, Yellowknife: Northwest Territories Government.

Northwest Territories Department of Education, Culture and Employment. (2016), Ministerial directive on inclusive schooling, Yellowknife : Northwest Territories Government.

Northwest Territories Government. (1995-2016). Loi sur l'éducation. Yellowknife : Northwest Territories Government.

Nouveau-Brunswick Department of Education. (2007). Gifted and talented students: A resource guide for teachers. Fredericton: NB Government.

Nouveau-Brunswick Department of Education. (2013). Policy 322 inclusive education. Fredericton: NB Government.

Nova Scotia Department of Education. (2011). L'éducation des élèves doués et le développement des talents. Halifax: NS Government.

Nova Scotia Government. (2008). Special education policy. Halifax: Department of Education and Early Childhood Development.

Nova Scotia Government. (1995-2015). Education Act. Halifax: NS Government.

Nunavut Department of Education. (2008). Foundation for inclusive education Inuglugijaittuq in Nunavut Schools. Iqaluit: Nunavut Department of Education.

Nunavut Government. (2008). Education Act: A reference guide for the commission scolaire francophone and school principals. Iqaluit: Nunavut Department of Education.

Office of Disability Issues. (2007). The disability inclusion policy framework government's response to the Saskatchewan Council on disability issues' Disability Action Plan. Regina: Government of Saskatchewan.

Poirier, D., Goguen, L. et Leslie, P. (1988) Education rights of exceptional children: A national study of multilevel commitment. Vancouver: Carswell.

Prince Edward Island. (1993-2013). School Act. Charlottetown: Minister of Education and Lifelong Learning of Prince Edward Island.

Rousseau, N., Dionne, C., Vézina, C. et Drouin, C. (2009). L'intégration scolaire d'enfants ayant une incapacité : perceptions des parents québécois. Revue canadienne de l'éducation. 32 (1) p. 34-59.

Saskatchewan Government. (1995). The Education Act. Regina: Government of Saskatchewan.

Saskatchewan Learning. (2006). Policy, guidelines, and procedures for alternative education programs alternative Grade 10, 11, and 12. Saskatoon: Saskatchewan Government.

Saskatchewan Learning. (2006). Policy, guidelines, and procedures for functional integrated programs. Saskatoon: Saskatchewan Government.

Saskatchewan Learning. (2007). Policy and procedures for locally modified courses of study. Saskatoon: Saskatchewan Government.

Saskatchewan Ministry of Education. (2011). Actualizing a needs-based model to support student achievement: A journey to transformation. Saskatoon: Ministry of Education.

Smith, C. M. (dir.). (2006). Including the gifted and talented: Making inclusion work for more gifted and able learners. Taylor \& Francis.

Sternberg, R. J. (2012). Intelligence. Dialogues in Clinical Neuroscience, 14, 19-27.

Sternberg, R. J. et Davidson, J. E. (2005). Conceptions of giftedness (2e éd.). New York, NY: Cambridge University Press.

Tremblay, P. (2018). Les plans d'intervention au Canada : analyse comparée des fonctions. Comparative and International Education/Éducation comparée et internationale. 47(1), 1-17.

Tremblay, P. (2021). L'identification des élèves à besoins spécifiques au Canada : entre catégorisation et noncatégorisation. Dans R. Malet et L. Baocun (dir.) Politiques éducatives et justice sociale. Perspectives internationales (p. 111-138). Berlin, Allemagne: Peter Lang Verlag.

Tremblay, P. et Belley, S. (2017). Les limites à la scolarisation en classe régulière au Canada : une analyse comparative. Revue éducation comparée. 18(2), 87-118.

Westwood, P. (2015). Commonsense methods for children with special educational needs. Routledge: Oxon. 
White, K., Fletcher-Campbell, F. et Ridley, K. (2003). What works for gifted and talented pupils: A review of recent research. Slough, Berkshire: National Foundation for Educational Research.

Yewchuk, C. (2000). Gifted education in Canada. The quest for giftedness: Proceedings of the 11th annual SAGE conference (p. 29-35). Calgary: Society for the Advancement of Gifted Education

Philippe Tremblay Aujourd'hui professeur en sciences de l'éducation à l'Université Laval à Québec, Philippe Tremblay a longuement travaillé en Belgique, d'abord comme instituteur primaire en enseignement ordinaire et spécialisé, puis comme chercheur et assistant à l'Université Libre de Bruxelles. Il est l'auteur de nombreuses recherches et articles portant sur le coenseignement et sur l'évaluation de l'enseignement spécialisé et de l'inclusion scolaire.

Jeanne Verreault-Tremblay est étudiante à la maitrise en Sciences de l'Éducation à l'Université Laval et enseignante au CEGEP. Également orthopédagogue de formation, elle travaille actuellement sur les relations entre coenseignants et élèves en contexte de classe pratiquant un coenseignement intensif en enseignement secondaire. 


\section{Annexe 1 - Tableau des mesures éducatives}

Tableau 3 : Liste des mesures éducatives visant les enfants doués, par province

\begin{tabular}{|c|c|c|c|c|c|}
\hline & Mesures de placement & Mesures d'accélération & $\begin{array}{c}\text { Mesures d'enseignement } \\
\text { différencié }\end{array}$ & Mesures extrascolaires & Autres \\
\hline 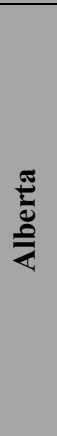 & $\begin{array}{ll}1 . & \begin{array}{l}\text { Écoles ou classes } \\
\text { spécialisées }\end{array} \\
\text { 2. } & \text { École à charte } \\
\text { 3. } & \begin{array}{l}\text { Programme } \\
\text { d'éducation }\end{array} \\
& \text { internationale (PEI) } \\
\text { 4. } & \text { Cours enrichis } \\
\text { 5. } & \begin{array}{l}\text { Regroupement par } \\
\text { habiletés }\end{array} \\
\text { 6. } & \text { Cours par } \\
& \text { correspondance } \\
\text { (programme scolaire) }^{5}\end{array}$ & $\begin{array}{ll}\text { 1. } & \text { Inscriptions doubles } \\
\text { ou simultanées } \\
\text { 2. } & \text { Accélération totale } \\
\text { 3. } & \text { Accélération partielle }\end{array}$ & $\begin{array}{ll}\text { 1. } & \text { Progression } \\
\text { continuelle } \\
\text { 2. } & \text { Différenciation } \\
\text { 3. } & \text { Séminaires } \\
\text { 4. } & \text { Sous-groupe de } \\
\text { besoins } \\
\text { 5. }\end{array}$ & $\begin{array}{ll}\text { 1. } & \text { Cours par } \\
\text { correspondance (hors } \\
\text { programme scolaire) }\end{array}$ & $\begin{array}{l}\text { 1.Bourses anglaises / } \\
\text { françaises }\end{array}$ \\
\hline 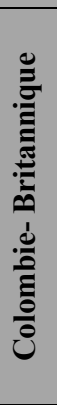 & $\begin{array}{ll}\text { 1. } & \begin{array}{l}\text { Ecoles ou classes } \\
\text { spécialisées }\end{array} \\
\text { 2. } & \begin{array}{l}\text { Regroupement par } \\
\text { habiletés }\end{array} \\
\text { 3. } & \begin{array}{l}\text { Programme } \\
\text { d'éducation }\end{array} \\
& \text { internationale (PEI) } \\
\text { 4. } & \text { Cours enrichis } \\
\text { 5. } & \text { Cours par } \\
& \text { correspondance } \\
\text { (programme scolaire) }\end{array}$ & $\begin{array}{ll}\text { 1. } & \text { Accélération partielle } \\
\text { 2. } & \text { Placement avancé }\end{array}$ & $\begin{array}{ll}\text { 1. } & \text { Différenciation } \\
\text { 2. } & \text { Études autonomes } \\
\text { 3. } & \text { Centres } \\
& \text { d'apprentissage } \\
\text { 4. } & \text { Programme compacté } \\
\text { 5. } & \text { Études autonomes } \\
\text { 6. } & \text { Mentorat } \\
\text { 7. } & \text { Sous-groupe de } \\
& \text { besoins }\end{array}$ & $\begin{array}{ll}\text { 1. } & \text { Cours par } \\
\text { correspondance (hors } \\
\text { programme scolaire) }\end{array}$ & $\begin{array}{ll}1 . & \text { Bourses } \\
\text { anglaises/françaises }\end{array}$ \\
\hline 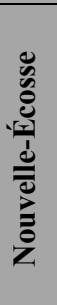 & $\begin{array}{ll}1 . & \begin{array}{l}\text { Programme } \\
\text { d'éducation }\end{array} \\
\text { internationale (PEI) } \\
\text { 2. } & \text { Regroupement par } \\
\text { habiletés } \\
\text { 3. } & \begin{array}{l}\text { Cours par } \\
\text { correspondance } \\
\text { (programme scolaire) }\end{array}\end{array}$ & $\begin{array}{ll}\text { 1. } & \text { Placement avancé } \\
\text { 2. } & \text { Inscriptions doubles } \\
\text { ou simultanées } \\
\text { 3. } & \text { Accélération partielle }\end{array}$ & $\begin{array}{ll}\text { 1. } & \begin{array}{l}\text { Sous-groupe de } \\
\text { besoins }\end{array} \\
\text { 2. } & \text { Études autonomes } \\
\text { 3. } & \begin{array}{l}\text { Centres } \\
\text { d'apprentissage }\end{array} \\
\text { 4. } & \text { Mentorat } \\
\text { 5. } & \text { Programmes } \\
& \text { parascolaires }\end{array}$ & $\begin{array}{ll}\text { 1. } & \text { Cours par } \\
\text { correspondance (hors } \\
\text { programme scolaire) }\end{array}$ & $\begin{array}{l}\text { Bourses anglaises/ } \\
\text { françaises }\end{array}$ \\
\hline
\end{tabular}

${ }^{5}$ Sans avoir directement été répertoriée dans les guides pédagogiques, la mesure de placement référant à la possibilité de suivre des cours par correspondance est néanmoins offerte dans les juridictions suivantes : la Colombie-Britannique, la Nouvelle-Écosse, l'Île-du-Prince-Édouard, le Manitoba, le Nunavut, la Saskatchewan, les Territoires du Nord-Ouest ainsi que le Yukon. Pour les autres juridictions, cette mesure est explicitement indiquée dans leurs guides respectifs. 


\begin{tabular}{|c|c|c|c|c|c|}
\hline 产 & 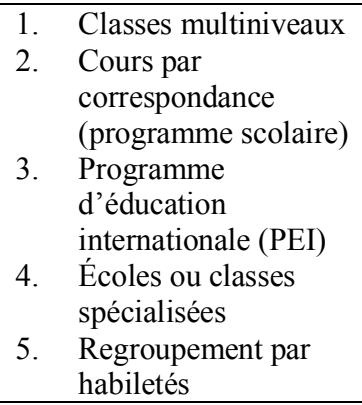 & $\begin{array}{ll}\text { 1. } & \text { Accélération totale } \\
\text { 2. } & \text { Accélération partielle } \\
\text { 3. } & \text { Cours par } \\
\text { correspondance } \\
\text { 4. } \\
\text { Inscription double ou } \\
\text { simultanée } \\
\text { 5. Placement avancé } \\
\text { 6. } \\
\text { Obtention de crédits } \\
\text { par la réussite d'un } \\
\text { examen } \\
\text { 7. }\end{array}$ & $\begin{array}{ll}\text { 1. } & \text { Programme compacté } \\
\text { 2. } & \text { Mentorat } \\
\text { 3. } & \text { Progression } \\
& \text { continuelle } \\
\text { 4. } & \text { Différenciation } \\
\text { 5. } & \text { Programmes } \\
& \text { parascolaires }\end{array}$ & $\begin{array}{ll}\text { 1. } & \text { Cours par } \\
\text { correspondance (hors } \\
\text { programme scolaire) }\end{array}$ & $\begin{array}{l}\text { 1. Bourses anglaises / } \\
\text { françaises }\end{array}$ \\
\hline 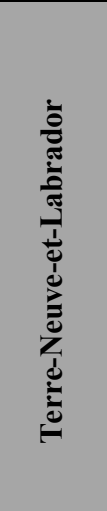 & $\begin{array}{ll}\text { 1. } & \text { Classes multiniveaux } \\
\text { 2. } & \text { Cours enrichis } \\
\text { 3. } & \text { Cours de courte durée } \\
\text { 4. } & \text { Mini cours } \\
\text { 5. } & \text { Cours par } \\
& \text { correspondance } \\
& \text { (programme scolaire) } \\
\text { 6. } & \text { Cours externes } \\
\text { 7. } & \text { Placement alternatif } \\
\text { 8. } & \text { Programme } \\
& \text { d'éducation } \\
\text { internationale (PEI) } \\
\text { 9. } & \text { Regroupement par } \\
& \text { habiletés }\end{array}$ & $\begin{array}{ll}\text { 1. } & \text { Accélération partielle } \\
\text { 2. } & \text { Accélération totale } \\
\text { 3. } & \text { Placement avancé } \\
\text { 4. } & \text { Classes multi- } \\
\text { niveaux }\end{array}$ & $\begin{array}{ll}\text { 1. } & \begin{array}{l}\text { Enseignement aux } \\
\text { habiletés réflexives }\end{array} \\
\text { 2. } & \text { Contrat } \\
& \text { d'apprentissage } \\
\text { 3. } & \text { Programme compacté } \\
\text { 4. } & \text { Différenciation } \\
\text { 5. } & \text { Études autonomes } \\
\text { 6. Étude de célébrités } \\
\text { 7. } & \text { Séminaires } \\
\text { 8. } & \text { Mentorat } \\
\text { 9. } & \text { Sous-groupe de } \\
& \text { besoins } \\
\text { 10. } & \text { Programmes } \\
& \text { parascolaires }\end{array}$ & $\begin{array}{ll}\text { 1. } & \text { Mini cours } \\
\text { 2. } & \text { Centres } \\
\text { d'apprentissage } \\
\text { 3. } & \text { The Education } \\
& \text { Program for Gifted } \\
\text { Youth (EPGY) } \\
\text { 4. } \begin{array}{l}\text { Cours par } \\
\text { correspondance (hors } \\
\text { programme scolaire) }\end{array}\end{array}$ & $\begin{array}{l}\text { 1. Bourses anglaises / } \\
\text { françaises }\end{array}$ \\
\hline 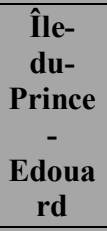 & $\begin{array}{ll}\text { 1. } & \begin{array}{l}\text { Cours par } \\
\text { correspondance } \\
\text { (programme scolaire) }\end{array} \\
\text { 2. } & \begin{array}{l}\text { Programme } \\
\text { d'éducation } \\
\text { internationale }(\mathrm{PEI})^{6}\end{array} \\
\end{array}$ & 1. Accélération totale & 1. Différenciation & $\begin{array}{ll}\text { 1. } & \text { Cours par } \\
\text { correspondance (hors } \\
\text { programme scolaire) }\end{array}$ & $\begin{array}{l}\text { 1. Bourses anglaises / } \\
\text { françaises }\end{array}$ \\
\hline 总 & $\begin{array}{ll}\text { 1. } & \begin{array}{l}\text { Cours par } \\
\text { correspondance } \\
\text { (programme scolaire) }\end{array} \\
\text { 2. } & \begin{array}{l}\text { Programme } \\
\text { d'éducation } \\
\text { internationale (PEI) }\end{array} \\
\end{array}$ & 1. Accélération totale & 1. Différenciation & $\begin{array}{ll}\text { 1. } & \text { Cours par } \\
\text { correspondance (hors } \\
\text { programme scolaire) }\end{array}$ & $\begin{array}{l}\text { 1. Bourses anglaises / } \\
\text { françaises }\end{array}$ \\
\hline
\end{tabular}

${ }^{6}$ Similairement aux cours par correspondance, sans avoir directement été répertorié dans les guides pédagogiques, le Programme d'éducation internationale (PEI) est une mesure de placement offerte à l'Île-du-Prince-Édouard, au Manitoba, au Nunavut, aux Territoires du Nord-Ouest ainsi qu'au Yukon. 


\begin{tabular}{|c|c|c|c|c|c|}
\hline हूँّ & 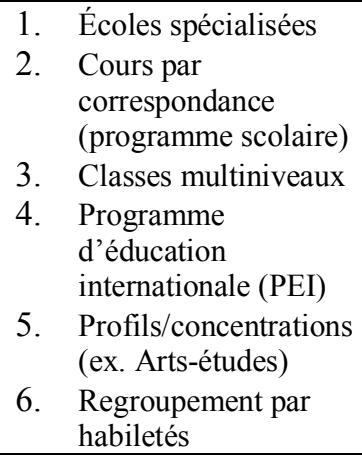 & $\begin{array}{ll}\text { 1. } & \text { Accélération totale } \\
\text { 2. } & \text { Classes multiniveaux } \\
\text { 3. } & \text { Entrée précoce } \\
\text { 4. } & \text { Saut de classe }\end{array}$ & $\begin{array}{ll}\text { 1. } & \text { Différenciation } \\
\text { 2. } & \text { Sous-groupe de } \\
\text { besoins } \\
\text { 3. Études autonomes } \\
\text { 4. } \\
\text { 5. } \\
\text { 6. Tutorat } \\
\text { Programmes } \\
\text { parascolaires }\end{array}$ & $\begin{array}{ll}\text { 1. } & \text { Cours par } \\
\text { correspondance (hors } \\
\text { programme scolaire) }\end{array}$ & $\begin{array}{l}\text { 1. Bourses anglaises / } \\
\text { françaises }\end{array}$ \\
\hline 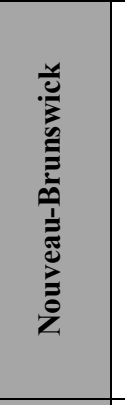 & $\begin{array}{ll}\text { 1. } & \begin{array}{l}\text { Programme } \\
\text { d'éducation } \\
\text { internationale (PEI) }\end{array} \\
2 . & \begin{array}{l}\text { Cours par } \\
\text { correspondance } \\
\text { (programme scolaire) }\end{array} \\
\text { 3. } & \text { Programmes enrichis }\end{array}$ & $\begin{array}{ll}\text { 1. Accélération totale } \\
\text { 2. Accélération partielle } \\
\text { 3. Placement avancé } \\
\text { 4. Inscription double ou } \\
\text { simultanée } \\
\text { 5. Obtention de crédit } \\
\text { par la réussite d'un } \\
\text { examen } \\
\text { 6. Cours par } \\
\text { correspondance }\end{array}$ & $\begin{array}{ll}\text { 1. } & \text { Sous-groupe de } \\
& \text { besoins } \\
\text { 2. } & \text { Programme compacté } \\
\text { 3. } & \text { Centre } \\
\text { d'apprentissage } \\
\text { 4. } & \text { Études autonomes } \\
\text { 5. } & \text { Mentorat } \\
\text { 6. } & \text { Séminaires } \\
\text { 7. } & \text { Différenciation } \\
\text { 8. } & \text { Programmes } \\
& \text { parascolaires } \\
\end{array}$ & $\begin{array}{ll}\text { 1. } & \text { Cours par } \\
\text { correspondance (hors } \\
\text { programme scolaire) }\end{array}$ & $\begin{array}{l}\text { 1. Bourses anglaises / } \\
\text { françaises }\end{array}$ \\
\hline 咅 & $\begin{array}{ll}\text { 1. } & \begin{array}{l}\text { Cours par } \\
\text { correspondance } \\
\text { (programme scolaire) }\end{array} \\
2 . & \begin{array}{l}\text { Programme } \\
\text { d'éducation }\end{array} \\
\text { internationale (PEI) }\end{array}$ & & 1. Différenciation & $\begin{array}{l}\text { 1. } \begin{array}{l}\text { Cours par } \\
\text { correspondance (hors } \\
\text { programme scolaire) }\end{array}\end{array}$ & $\begin{array}{l}\text { 1. Bourses anglaises / } \\
\text { françaises }\end{array}$ \\
\hline 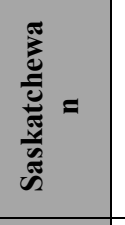 & $\begin{array}{ll}\text { 1. } & \begin{array}{l}\text { Programme } \\
\text { d'éducation }\end{array} \\
\text { internationale (PEI) } \\
2 . & \text { Cours par } \\
\text { correspondance } \\
\text { (programme scolaire) }\end{array}$ & & 1. Différenciation & $\begin{array}{l}\text { 1. Cours par } \\
\text { correspondance (hors } \\
\text { programme scolaire) }\end{array}$ & $\begin{array}{l}\text { 1. Bourses anglaises / } \\
\text { françaises }\end{array}$ \\
\hline 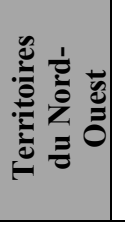 & $\begin{array}{ll}\text { 1. } & \begin{array}{l}\text { Programme } \\
\text { d'éducation }\end{array} \\
\text { internationale (PEI) } \\
\text { 2. } & \begin{array}{l}\text { Cours par } \\
\text { correspondance } \\
\text { (programme scolaire) }\end{array} \\
\end{array}$ & 1. Accélération totale & 1. Programme compacté & $\begin{array}{l}\text { 1. Cours par } \\
\text { correspondance (hors } \\
\text { programme scolaire) }\end{array}$ & $\begin{array}{l}\text { 1. Bourses anglaises / } \\
\text { françaises }\end{array}$ \\
\hline
\end{tabular}




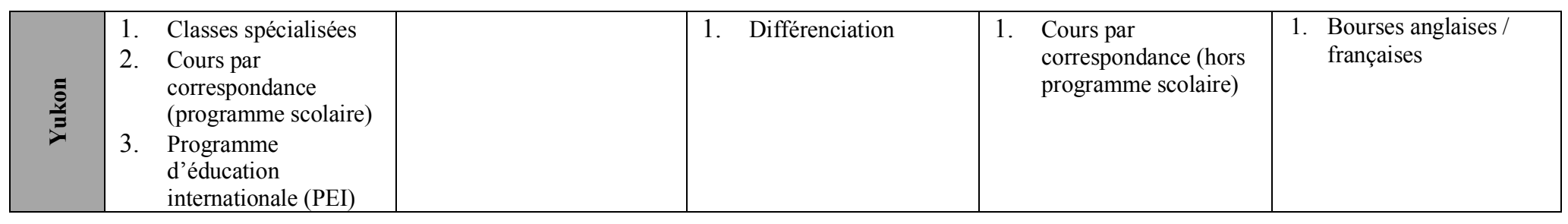

